\title{
Using Kinesthetic and Tactile Cues to Maintain Exercise Intensity
}

\author{
Aaron R. Ferber, Michael Peshkin, Member, IEEE, and J. Edward Colgate, Member, IEEE
}

\begin{abstract}
Haptic cues may be able to assist an individual who is engaged in a manual control task, freeing visual and auditory attention for other mental tasks. We describe an experiment in which subjects attempted to step at a consistent pace on a stair climber exercise machine, which was modified for haptic cuing through the legs. Subjects' visual attention was engaged by a video game. Five different haptic cues for consistent pacing were investigated, two of them more kinesthetic in nature and three that were more tactile. Results showed that haptic cues could indeed improve the manual control task performance without diminishing the visual attention task performance. The tactile cues generally outperformed the kinesthetic ones.
\end{abstract}

Index Terms-Haptics, haptic communications, user interfaces, manual control tasks, foot haptics

\section{INTRODUCTION}

A LTHOUGH manual control tasks are by nature physical acts, they can require constant mental attention. Task performance can suffer when mental distractions are present, and depending on the manual task at hand, the impact could range from frustrating to dangerous. Consider, for example, the situation of trying to read while exercising on an elliptical trainer or stair climber at a health club. Although some level of exertion might be maintained, it can be difficult to sustain a high workout intensity when engrossed with reading material.

This dilemma has been well considered by designers of exercise equipment, as evidenced by the wide array of products available to help users maintain workout intensity. These include immersive video [1], video games [2], [3], [4], simulated personal trainers [5], synchronized music [5], [6], [7], [8], "motivational interfaces" [9], and electronic "rewards" such as TV viewing [10], [11]. Some machines have "motivational programming" such as "Hill Climbing" that are intended to keep the user on a certain type of workout for a set period of time.

Powered machines, such as treadmills and some stair steppers, require a user to exercise at a pre-determined level or risk falling off the machine. This same "motivation by fear" is true of some non-powered machines as well, such as stair steppers on which the user must go fast enough to avoid the unpleasant "bottoming out" of the pedals. Although such systems may successfully keep users working out with high intensity, we do not believe that motivation by fear makes for an enjoyable experience, and it is not the same as encouraging or reminding a user to maintain intensity.

- A.R. Ferber is with IDEO, LLC, Evanston, IL 60201. E-mail: \{aferber@ideo.com\}

- M. Peshkin and J.E. Colgate are with the Department of Mechanical Engineering, Northwestern University, Evanston, IL 60201.

E-mail: \{peshkin, colgate\}@northwestern.edu

Manuscript received $x x x x$ 2008; revised $x x x x 200 x$.
The motivational systems mentioned previously cover a range of approaches, but they rely primarily on the visual and auditory channels. Yet it is typically visual and auditory attention that a user wants to divert elsewhere - perhaps to a book, to the television, or to a conversation. These motivational systems compete for the mental resources needed for such multitasking. None of these motivational systems take advantage of the physical interface that the user has with the exercise machine. However, humans can often respond to changes in a physical interaction with little thought or attention: a driver's grip tightens and maintains control of the steering wheel when her vehicle hits a pothole; a waiter corrects his balance and manipulates his fingers, wrist, and arm when an item shifts on his tray; vehicle occupants subconsciously lean into turns and forward accelerations. Note that these sensations are peripheral; they are not the focus of the receiver's attention. Perhaps this acute ability to respond to what we feel through kinesthetic and tactile sensations - even peripheral sensations - can be exploited by designers to help users better complete manual control tasks. These include tasks such as driving a vehicle, using exercise equipment, steering a pallet jack, operating assembly line equipment, or using rehabilitation equipment, to name a few. With regard to the exercise task, perhaps it is possible to design an exercise machine that haptically assists a user in maintaining a desired workout intensity in the face of mental distractions.

In order to design such systems, it is necessary to understand how information can be haptically communicated to the operator of the machine in a reliable and efficient manner while the control task is being undertaken - with all the distractions and haptic noise that come with it. In this paper, we explore this topic in the specific context of haptically assisting a user of a stair climber in maintaining exercise intensity.

Some of the material in this paper was documented in [12] and introduced in [13]. 


\section{BACKGROUND}

The design of haptic communications has been studied in a broad variety of contexts including haptics, product design, telerobotics, ergonomics, human-robot interaction, stroke rehabilitation, and psychology.

Human-computer interaction researchers have investigated using vibrating mice [14], [15], force-feedback styluses [16], [17], [18], tactile foot platforms [19], and other haptic devices to provide feedback for tasks such as desktop navigation [15], shared control of an application [16], [20], [21], or even social interaction [22], [23], [24]. These studies have shown that individuals can successfully discriminate between different values of vibration frequency [25], location of vibration sensation [19], rhythm [26], and multiple other factors that can define haptic communications. Several studies have shown that subjects can complete these haptic discrimination tasks even in the presence of mental distractions [14], [15], [25].

Commercial entities have shown interest in haptic communications including research related to mobile devices and automobiles. These studies have shown that haptic communications can be learned and recalled in a reasonable amount of time so as to be viable in products [27], [28]. They also show the variety of situations in which haptic communications can be used. With mobile devices, haptics have been used to notify users of information such as message type and call priority [26]. Automotive studies have shown that haptic sensations can communicate information such as navigational directions [28] and collision warnings [29], [30], [31].

There has been some work on the idea of haptic information flow between user and machine during shared control of a manual control task, most often studied within the realm of driving an automobile. These studies include haptic information received through the steering wheel [30], [32], [33] and through the pedals [34], [35]. These works have shown that providing a driver with haptic information by directly manipulating the dynamics of the control mechanisms can improve task performance while reducing visual and mental load.

There have been multiple studies around modifying exercise equipment to affect a user's workout in some manner. Li, Horowitz, and Shields have completed multiple studies on exercise machine control systems, such as designing a controller to maximize the mechanical power output of the user throughout the exercise motion so that a given level of exercise can be achieved in less time [36], [37], [38], [39], [40], [41]. In another study, the step resistance of a stair stepper is controlled such that the user's step rate is held constant [42], [43]. Kazerooni and He built an active 2-DOF arm exercise machine that allows the user to interact with virtual systems such as a mass/spring/damper [44]. Similarly, Zhang et al. developed algorithms to control a motor with a handle on it such that it served as an arm exercise machine that optimized user power input [45]. Motamarri et al. also developed a motor controller for an

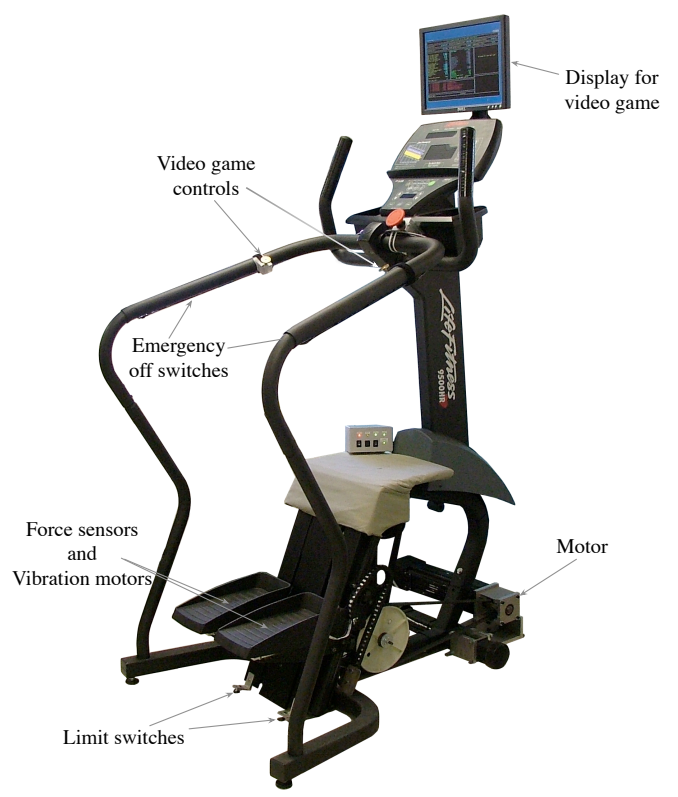

Fig. 1. The haptic stair climber

exercise machine that provides resistance or assistance to the user to achieve some goal workout based on an "expert model" [46]. Hayward and Hollerbach used a harness around the torso of subjects walking on an inclined treadmill to simulate the feeling of walking on stairs [47]. Finally, Minetti et al. developed a feedbackcontrolled treadmill that automatically adjusted its speed to the desired speed of the user [48].

Also relevant to our study is work on the design of dual task experiments, such as Multiple Resource Theory. Wickens explores the time-sharing efficiency of two tasks defined by their use of mental resources such as sensory modality and processing stage [49]. In short, the more two tasks require the same resources, the less efficient the time-sharing will be. Wickens' work also includes a summary of other factors that have been found to affect time-sharing efficiency.

\section{Study OVERVIEW}

We modified a commercial stair climber so that it could serve as a platform for developing and testing a wide range of haptic sensations (Section 4). We then used this platform to develop a sizable library of haptic communications that encourage a user to step at a given step rate. From this library, we selected a promising subset of haptic communications to test experimentally (Section 5). This subset was then quantitatively and qualitatively evaluated with thirty-six subjects (Section 6).

\section{THE STAIR CLIMBER}

We modified a LifeFitness 9500HR stair climber, shown in Fig. 1, such that much of the user interaction experience could be controlled. The pedal resistance and return mechanisms were removed and replaced with a 
Kollmorgen Goldline brushless motor (model ME2-207B-A2-B3P3D, max speed $2800 \mathrm{rpm}$, peak torque $12 \mathrm{~N} \cdot \mathrm{m}$ ) connected to the pedals via a pulley system and controlled using a ServoToGo board in a computer running QNX real-time operating system. Since the motor had complete control over pedal position, it could provide both gross pedal movement and haptic communications overlaid on the gross movement. (Such haptic communications will be described in Section 5.)

\subsection{Sensors and switches}

Multiple sensors and switches were installed on the stair climber to provide data acquisition, control feedback, and safety. An optical encoder, integral to the motor, provided high-precision motor position data, and a linear potentiometer attached to a pedal provided pedal position data. Limit switches were installed under each pedal, and the user was given manual off switches in the form of two TapeSwitches, one on the bottom of each handrail. If the user needed to stop the machine, he could simply squeeze a handrail. Finally, four load cells were installed between each pedal and pedal platform, providing user force input data (resolution $+/-5 \mathrm{~N}$ ).

\subsection{Video game interface}

To provide our mental distraction task, we installed a custom video game interface that the user could safely operate while using the stair climber. This consisted of a display mounted to the stair climber at the user's eye level and a 10-turn potentiometer on each of the handrails, located such that the user could turn the potentiometer with his thumb while still holding the handrails. The task, described in Section 6.1, is similar to the video game Pong. ${ }^{\mathrm{TM}}$

\subsection{Pedal vibration motors}

The stair climber motor can provide low-frequency and impulsive forces to the user, and we additionally installed a cell phone vibration motor under each pedal to provide higher frequency $(140 \mathrm{~Hz})$, low amplitude vibrations to the feet. The haptic signal from these motors was very noticeable on the feet even when wearing shoes.

\subsection{Virtual flywheel base model}

The movement of the stair climber pedals was generally dictated by the virtual model of the flywheel shown in Fig. 2. System parameters, as denoted in Fig. 2, were set at $\mathrm{I}=6.0 \mathrm{~kg} \cdot \mathrm{m}^{2}$ and $\mathrm{r}_{p}=0.1 \mathrm{~m}$ (providing a step height of $0.2 \mathrm{~m}$ ). Flywheel damping torque was calculated as $\tau_{d}=b \cdot \omega$, with $b=2.0 \mathrm{~N} \cdot \mathrm{m} \cdot \mathrm{s}$.

This model serves as a good base model for several reasons. First, the equations dictating pedal motion can be derived directly from the physical analog of the flywheel, leading to a simple control algorithm with a "natural" feel for the user. Second, resistance felt by the

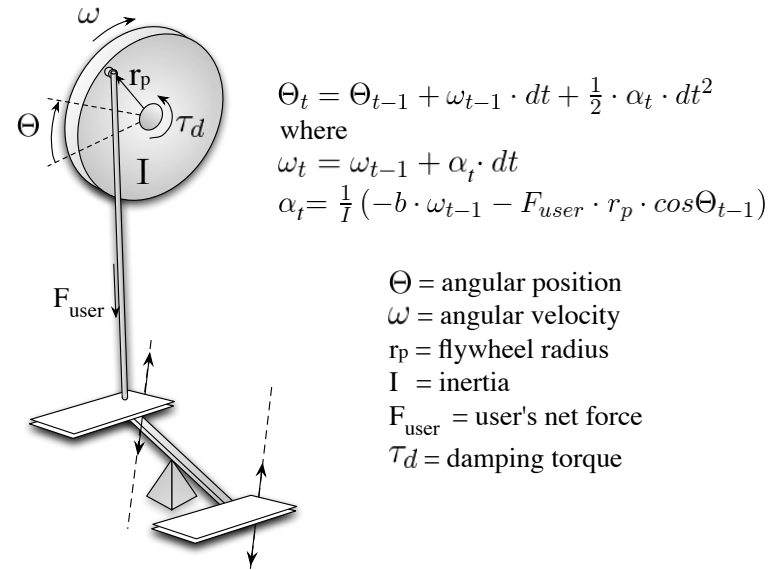

Fig. 2. Virtual flywheel base model

user can be controlled by the single parameter $b$, the coefficient of damping. Finally, the flywheel provides the user with a sensation of momentum due to its virtual inertia, I, which mimics the inertia that one has when running up a real flight of steps. We believe this sensation makes exercise on the stair climber a more satisfying experience.

\section{HAPTIC COMMUNICATIONS}

A variety of haptic communications were developed for the stair climber. The intent of these communications is to encourage the user to step at a given pace without forcing him to do so. There are many types of possible haptic communications, and many sub-types within each type. In developing our library, we spanned a range of approaches to haptic communications rather than focusing on just a few or trying to be exhaustive. We consider this reasonable because the use of haptic communications during the type of manual control task we are studying has seldom been explored in-depth previously, and therefore starting with a wide range of haptic communication types is appropriate at this stage.

Although there are many ways to categorize haptic communications, one way is whether they are more kinesthetic or tactile in nature. The more kinesthetic communications of our library are provided ${ }^{1}$ in Table 1 and the more tactile ones in Table 2. (For brevity, the communications are referred to as cues in these tables and in subsequent text.) In the descriptions of the haptic cues, the user's speed is defined as the rotational velocity of the flywheel, $\omega$, and the goal speed is defined as $\omega_{\text {goal }}$. "Going slower than $\omega_{\text {goal }}$ " is defined as going $98 \%$ or less of $\omega_{\text {goal }}$. This definition allows for small, shortterm fluctuations in user speed. Such fluctuations are permissible, as the intent is really to keep the moving average of $\omega$ at or above $\omega_{\text {goal }}$.

1. These cues are implemented using multiple logic arguments and do not lend themselves to brief mathematical notation. Therefore we have explained them qualitatively here and have made the code available online as supplemental material to this manuscript. 
TABLE 1

Kinesthetic haptic cues

Cue name Description
Selected Reason
E-Boost The user is given a very slight increase in speed if he starts stepping too slowly. The strength of the boost is such that it is noticeable to the user but not quick enough to injure him, and such that the user could still come to a stop if desired, achieved by having a delay of several seconds between boosts. The boost only speeds up the user slightly, not necessarily all the way to $\omega_{\text {goal }}$.

Inertia The inertia of the virtual flywheel changes as a function Lock of $\omega$, as shown in Fig. 3. It is a maximum at $\omega_{\text {goal }}$ and decreases to a minimum value above and below this value. The intent is that once the user is stepping at $\omega_{\text {goal }}$, it would be difficult to change speed due to the increased inertia at this speed.

Asymmetric Simon et al. [50] conducted a study in which subjects resistance using a leg press encountered resistance that varied in proportion to the symmetry with which they were pushing on each foot. They found that users could subconsciously adjust the symmetry in their push so as to minimize the resistance encountered. If the converse is true as well, users might be able to react to an asymmetry in the force encountered by each leg. Thus, in this cue, users encounter an asymmetry in the resistance on each leg that grows proportional to $\left|\omega_{\text {goal }}-\omega\right|$, up to a maximum. In our implementation, resistance increases on the left leg and decreases on the right. By subjectively setting the desired feel, the increase on the left happened to be greater than the decrease on the right, resulting in a net increase in resistance.

Flywheel The virtual flywheel is connected through a virtual connected damper to a virtual motor. The virtual motor is driven to a motor at a speed such that the user feels a pull to go faster if he is going slower than $\omega_{\text {goal }}$ and feels increased resistance if he is going faster than $\omega_{\text {goal }}$.

Geared A set of virtual gears exists between the pedals and the flywheel virtual flywheel. If the gear ratio is increased above 1:1, the user encounters increased resistance and the virtual flywheel begins to spin faster than the user is stepping, storing up virtual kinetic energy. If the gear ratio is then reduced, the virtual kinetic energy contained in the virtual flywheel transfers to the user, giving a sensation of being pulled faster. This cue was implemented such that the gear ratio was 1:1 while the user stepped at $\omega_{\text {goal }}$. If the user began to slow down, the gear ratio was sinusoidally increased to $1.6: 1$ and then returned to $1: 1$ over a period of $\sim 6$ seconds.

Damping In this cue, the amount of damping on the flywheel is a torque function of the user's speed, as shown in Fig. 4. If a user valley is stepping at a speed slightly slower than $\omega_{\text {goal }}$ and begins to step slightly faster, the resistance will decrease and, assuming that he continues to push with the same force, he will continue to go even faster. If he begins to step faster than $\omega_{\text {goal }}$, he will encounter increased resistance and, assuming he continues pushing with the same force, the increased resistance will slow his acceleration. The net effect is a passive system that keeps the user stepping near $\omega_{\text {goal }}$.

$\checkmark \quad$ The E-Boost cue was found to have a subtle and pleasing kinesthetic feel while also logically conveying a message of "speed up". The sensation is reminiscent of a slight push from behind, a gentle nudge to go faster. some trials of this cue, test subjects unconsciously sped up if the valley was dynamically shifted to the right (thereby increasing $\omega_{\text {goal }}$ ). Of course, these could have been coincidental occurrences. In other trials, users said that they could feel the increased resistance on either side of $\omega_{\text {goal }}$, but they could not determine if their step rate was too fast or too slow. This is a consequence of users trying to consciously abide by their understanding of the cue, while the cue was intended to subconsciously keep them stepping at $\omega_{\text {goal }}$. 
TABLE 2

Tactile haptic cues

$\begin{array}{lll}\text { Cue name Description } & \text { Selected Reason }\end{array}$

\begin{abstract}
Tapping By adding a very quick, brief acceleration to the pedal movement four times a second, this cue gives the user a tapping sensation on his feet. The tapping is activated when the user steps at a speed slower than $\omega_{\text {goal }}$ and turns off when $\omega_{\text {goal }}$ is reestablished.
\end{abstract}

Metronome This cue taps on the user's feet at the same rate he should be stepping to achieve $\omega_{\text {goal }}$. The tapping never shuts off, serving as a constant, potentially subconscious aide in maintaining $\omega_{\text {goal }}$.

Doubletime This cue is similar to the metronome cue mentioned metronome above, but taps are given at a "double-time" rate.

Variable The user feels a tap at the bottom of each step if location stepping at $\omega_{\text {goal }}$. If the user steps at a rate less than tapping $\omega_{\text {goal }}$, the tap will be felt before reaching the bottom, and if he steps faster than $\omega_{\text {goal }}$, the tap will be felt after reaching the bottom.

Variable Tapping occurs constantly at $4 \mathrm{~Hz}$, but the amplitude amplitude of the taps is proportional to $\left|\omega_{\text {goal }}-\omega\right|$. Thus, if tapping a user steps at $\omega_{\text {goal }}$, he feels no tapping, and tap intensity increases with deviation from $\omega_{\text {goal }}$.

Vibration

The small vibration motors mounted to the underside of each pedal provide a vibration sensation $(140 \mathrm{~Hz})$ on both feet if the user is walking too slow and turns off when $\omega_{\text {goal }}$ is reestablished.

Vibrating Since humans are able to detect changes in sensations pulses better than they are able to detect a constant sensation [54], this cue turns the vibration motor on and off at $2 \mathrm{~Hz}$ when the user is stepping slower than $\omega_{\text {goal }}$.

Variable The vibration motors are always "on", but the voltage intensity to the motors is proportional to $\left|\omega_{\text {goal }}-\omega\right|$. Thus, vibration the user will feel a more intense vibration as deviation from $\omega_{\text {goal }}$ increases.

A subset of cues was selected to be tested experimentally. The selection process included considerations for material found in relevant literature, feedback from pilot test subjects, and practical concerns of implementation and safety. They were also intentionally selected to span a variety of approaches. Tables 1 and 2 indicate whether each cue was selected for testing and the reason for that decision. In total, two kinesthetic cues (E-Boost and Asymmetric Resistance) and three tactile cues (Tapping, Doubletime Metronome, and Vibration) were selected.

\section{THE EXPERIMENT}

An experiment was developed to quantitatively and qualitatively test the five selected haptic cues. In this experiment, subjects were given the task of stepping at
The tapping cue sent a very clear, noticeable message to the user and did not actively interfere with the user's desired stepping behavior.

$\times \quad$ Preliminary test subjects reported that, because the tapping was occurring so close to their stepping rate when they were very near $\omega_{g o a l}$, it was difficult to determine if they were stepping slightly too fast or slightly too slow.

$\sqrt{ } \quad$ Unlike Metronome, testers found it fairly easy to step in sync with this cue, perhaps even subconsciously. This is not surprising since humans are profoundly good at synchronizing with a beat [51].

$\times \quad$ Preliminary test subjects could not detect the change in position with enough resolution to render this cue effective.

$\times \quad$ This cue did not provide enough resolution for the preliminary test subjects to accurately calibrate their speed by it. That humans do not have high precision in detecting variations in haptic amplitude is well documented in the literature [26], [52], [53].

$\sqrt{ } \quad$ The vibration cue could be thought of as the same interaction as the Tapping cue with simply a different amplitude and frequency, and this cue proved effective in similar fashion to the Tapping cue.

$\times \quad$ The vibrating pulses cue seemed noticeable and unobtrusive. However, since it was very similar to the Tapping and Vibration cues, it was not selected for further testing.

$\times \quad$ As with the Variable amplitude tapping cue, pilot test subjects had a difficult time detecting changes in vibration intensity.

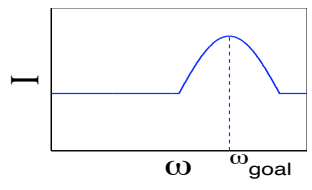

Fig. 3. $I-\omega$ relationship for the Inertia Lock cue

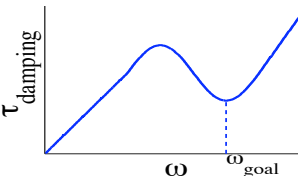

Fig. 4. $\tau_{d}-\omega$ for Damping torque valley cue a workout pace as consistently as possible. Additionally, they were to complete the secondary task of playing a video game. For part of the time that they were doing this multitasking, they were provided one of the haptic cues to assist them in stepping at their goal speed, and for part of the time they were given no such cue. Task performance with and without the haptic cue could then be compared. This experiment is explained in more detail in the following subsections. 


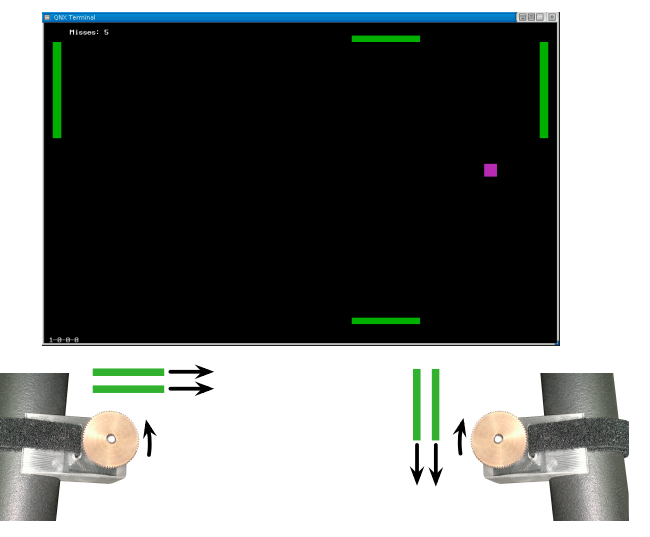

Fig. 5. Video game screen and control knobs

\subsection{The secondary task}

For the secondary task, we desired a task that required continuous attention, so as to prevent the subjects from switching attention between primary and secondary tasks; provided a single metric score; was entertaining so as to naturally engage the subject; and could be safely completed while operating the stair climber.

The secondary task developed was a simple Pong ${ }^{\mathrm{TM}}$ type of video game. In this video game, shown in Fig. 5, users attempt to keep a bouncing ball from leaving the screen by blocking it with "paddles". These paddles were controlled by scroll wheels, one on each handrail, which users could turn using their thumbs. The controls, shown in Fig. 5, were made intentionally unintuitive (according to pilot test subject input) so as to require the most continuous attention. Pilot test subjects also helped establish a ball speed that was reasonable to control while still requiring continuous attention ( $\sim 10 \mathrm{in} / \mathrm{sec})$. The user's score on this secondary task is the number of times the ball gets past the paddles ("misses").

Considering our tasks in the context of Wickens' work [49], we expect these tasks to cause time-sharing conflict for our subjects because both require manual response and spatial processing codes, have different timing requirements, different control dynamics, and similar directionality (both involve vertical motion).

\subsection{Experiment trial timeline}

Each subject completed five trials, one for each haptic cue. Each trial lasted two minutes and consisted of four time periods, as shown in Fig. 6. First, the 30-second warm-up period allowed subjects time to get up to speed and settle into a consistent pace. The next 30 seconds comprised the "baseline" period during which the subject was only completing the consistent stepping task. Near the end of this period, the computer calculated the subject's average speed. This "baseline speed" served as the goal speed, $\omega_{\text {goal }}$, during the subsequent two periods. That is, the goal is for the user to continue stepping at the same "fast workout pace". Five seconds before the baseline period ended, the screen beeped and displayed

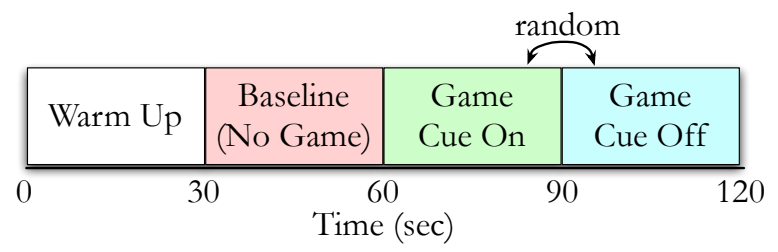

Fig. 6. Experiment timeline

a message that the subject should prepare to begin the video game. During pilot tests, this message caused subjects to vary their speed, presumably because their focus shifted away from the stepping task. For this reason, the goal speed mentioned previously was calculated three seconds before this message was displayed.

During the next two time periods, the subject was completing both the consistent stepping task and the video game secondary task. The haptic cue was present during one of these time periods, selected randomly. At the end of the trial, the screen would beep and display a message that the trial was finished, and the stair climber would slow to a stop.

\subsection{Experiment Execution}

After clearing the experiment with the Institutional Review Board, thirty-six subjects were recruited using flyers posted on the university campus. Subjects were required to be between the ages of 18 and 30 and in good health, and they were compensated $\$ 20$ for participating. There were 14 female and 22 male subjects with an average age of 22, standard deviation 2.95 .

Subjects were given a verbal explanation of the stair climber's features and operation, and the experimental protocol was explained. In this protocol, we instructed subjects to step at a "fast workout pace" ${ }^{2}$ as consistently as possible. We told them that stepping consistency was their goal for the first minute. After one minute, a video game would appear onscreen, and they should continue stepping at the same workout pace while playing the video game. The tasks of stepping consistency and video game performance were described to be of equal importance. Finally, subjects were told that at some time while they were playing the video game, they might feel the machine providing them with a physical sensation to help them maintain their consistent pace. The haptic cue for each trial was explained just before the trial started. We explained the cues because we are interested primarily in the efficacy of each haptic cue in assisting subjects rather than the learnability of each cue.

Before beginning the five trials, subjects were asked to play the video game while standing still on the stair climber. The first 30 seconds gave them a chance to understand the game, and then their score was recorded for the next 30 seconds to serve as a baseline game score.

2. In preliminary tests, we saw more variability in speed caused by the secondary task when subjects were stepping at a faster, "running" pace. This could relate to the fact that, in running, energy efficiency is relatively insensitive to speed [55]. 


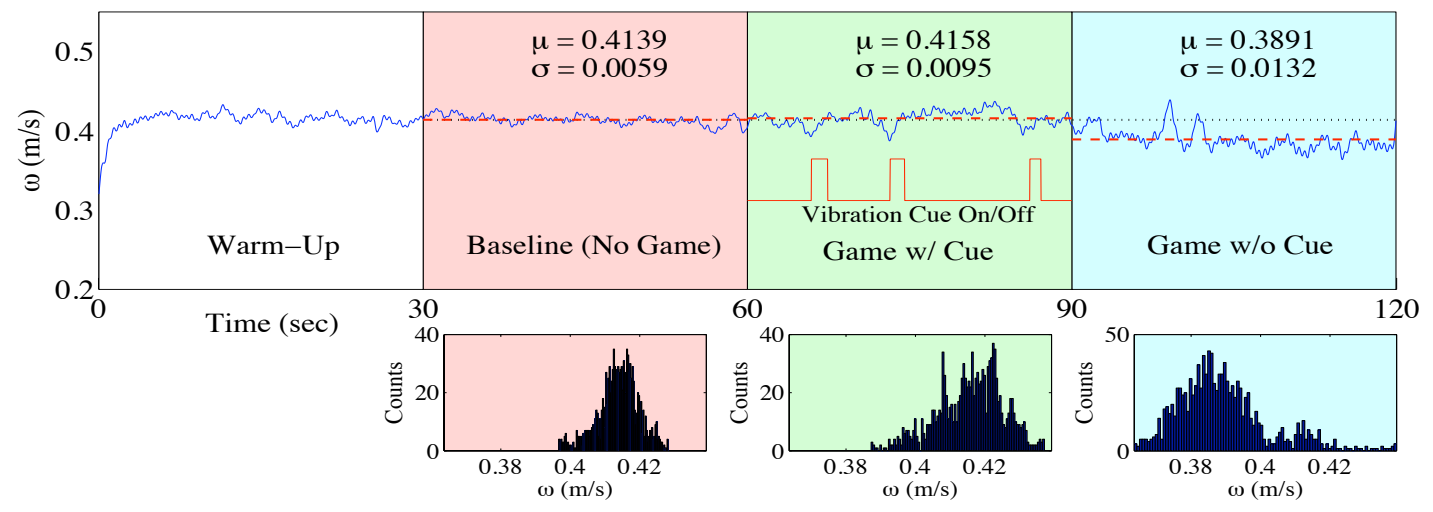

Fig. 7. Hypothetical subject data showing visualizations of stepping consistency metrics $\mu$ (average speed, visualized as the dashed line) and $\sigma$ (speed standard deviation, visualized in the histogram beneath each period)

Each subject performed five trials, one for each haptic cue. The presentation order of haptic cues was randomized. The computer controlling the stair climber recorded data on each trial including step rate and video game performance. After each trial was completed, the subject rested and completed a questionnaire about her performance and perception of the haptic cue.

In addition to the controls mentioned previously, several other controls were put in place. Trial duration was intentionally kept rather brief ( 2 minutes total) in order to avoid affects of fatigue and to keep total experiment duration to a reasonable amount of time. Subjects were also provided with a bottle of water and were allowed to take an unlimited break between trials in order to avoid effects of fatigue on the data. A sheet was hung around the front and sides of the stair climber to minimize visual distractions, and subjects wore headphones playing pink noise to minimize auditory distractions. This pink noise also helped to prevent subjects from using the noise of the machine to keep a consistent pace.

More details of the protocol can be found in [12].

\subsection{Data analysis}

Many quantitative variables were recorded during the experiment trials. The primary questions to be addressed by this data are how consistently subjects kept their speed and how well they performed on the video game. To evaluate the former of these questions, we developed a set of metrics which we will explain using data from a hypothetical subject, shown in Fig. 7.

A filtered ${ }^{3}$ version of the hypothetical subject's speed ${ }^{4}$ is plotted in the top graph of Fig. 7. The subject gets up to speed during the warm-up period and settles into a cadence with a mean speed of $0.4139 \mathrm{~m} / \mathrm{s}$ during the baseline period. This speed is held fairly steady, with a standard deviation of $0.0059 \mathrm{~m} / \mathrm{s}$. These mean $(\mu)$

3. Filtered with an acausal, low-pass filter, bandwidth $2 \mathrm{~Hz}$.

4. Stepping speed could be presented in a variety of units, such as $\mathrm{rad} / \mathrm{s}$ of the flywheel, steps/minute, or meters/second climbed by the user. We use meters/second as it is the most commonly used metric for stair climbers in exercise research. and standard deviation $(\sigma)$ values are listed at the top of the baseline period and are visualized graphically as a dashed line spanning the baseline region and a histogram under the baseline period, respectively.

Just before the video game turned on, the computer assigned the subject's baseline average speed of $0.4139 \mathrm{~m} / \mathrm{s}$ to be the goal velocity, $\omega_{\text {goal }}$. This is shown as a dotted line spanning the last two time periods of the plot. As the subject begins to play the game (starting at 60 seconds), his velocity has more variation than it did during the baseline period. However, when his speed drops below the haptic cue threshold of $98 \%$ of $\omega_{\text {goal }}$, the haptic cue (in this case, the Vibration cue) turns on. This can be seen in the "Game w/ Cue" period, immediately underneath the velocity plot. In theory, the Vibration cue notifies the subject to speed up, and once the subject brings his speed above $98 \%$ of $\omega_{\text {goal }}$, the haptic cue shuts off. In this example, the subject slows down and encounters the haptic cue three times.

Starting at 90 seconds, however, the haptic cue was no longer available. During this last period, the subject slows down to a mean speed of $0.3891 \mathrm{~m} / \mathrm{s}$ and the standard deviation increases to $0.0132 \mathrm{~m} / \mathrm{s}$.

After calculating the speed mean and standard deviation for each period of the hypothetical subject's data, we calculate the change $(\Delta)$ in these values between the baseline period and the video game periods. That is, we find the difference in speed mean and speed standard deviation between 1) the baseline and when the game was on with the haptic cue, and 2) the baseline and when the game was on without the haptic cue. The change in the hypothetical subject's mean speed was

$$
\begin{aligned}
\Delta \mu_{\text {cue_on }} & =\mu_{\text {cue_on }}-\mu_{\text {Baseline }} \\
& =0.4158-0.4139=+0.0019 \mathrm{~m} / \mathrm{s}
\end{aligned}
$$

when playing the game with the haptic cue on and

$$
\begin{aligned}
\Delta \mu_{\text {cue_off }} & =\mu_{\text {cue_off }}-\mu_{\text {Baseline }} \\
& =0.3891-0.4139=-0.0248 \mathrm{~m} / \mathrm{s}
\end{aligned}
$$

when playing the game without the haptic cue, compared to his speed during the baseline period. Similarly, 
the change in standard deviation of the speed was

$$
\begin{aligned}
\Delta \sigma_{\text {cue_on }} & =\sigma_{\text {cue_on }}-\sigma_{\text {Baseline }} \\
& =0.0095-0.0059=+0.0036 \mathrm{~m} / \mathrm{s}
\end{aligned}
$$

when playing the game with the haptic cue on and

$$
\begin{aligned}
\Delta \sigma_{\text {cue_off }} & =\sigma_{\text {cue_off }}-\sigma_{\text {Baseline }} \\
& =0.0132-0.0059=+0.0073 \mathrm{~m} / \mathrm{s}
\end{aligned}
$$

when playing the game without the haptic cue, compared to his speed's standard deviation during the baseline period.

The change in average speed $(\Delta \mu)$ provides a measure of how well the subject kept the same speed between periods, which we call inter-period consistency. Change in standard deviation $(\Delta \sigma)$ gives a measure of how evenly the subject kept that speed within the period, which we call intra-period consistency. We can compare these metrics between the cue on and cue off periods to determine whether the haptic cues helped the subject to step more consistently.

This analysis method is valid for most of the haptic cues, but it is not valid for understanding the effect of the E-Boost cue because this cue directly changes the user's velocity. However, by looking at the metric of the subject's power input (Power $=F \cdot \omega$, where $F$ is the user's force input), we can see whether an increase in speed when the cue is on is attributable to the subject. Following the same analysis described previously for speed, the metrics of $\Delta \mu_{\text {power }}$ and $\Delta \sigma_{\text {power }}$ can be calculated.

Once all speed and power $\Delta \mu^{\prime}$ s and $\Delta \sigma^{\prime}$ s were calculated for all valid trials, the $\Delta^{\prime}$ s were averaged across all trials for each cue. Matched-pair t-tests were performed between the metrics for cue on and cue off for each cue of each subject to determine whether there existed a statistically significant difference between the two.

We also analyzed the recorded data to determine if the haptic cues interfered with the task of playing the video game. We found the average number of "misses" on the game during each period of each trial and performed a matched-pairs t-test to determine if a statistically significant difference existed between the number of misses when the haptic cue was on and when it was off.

\section{Results}

The thirty-six subjects provided a total of 180 trials. Not every trial resulted in a valid data set, though, due to one of the following reasons:

- Although every subject completed all five trials, not every subject encountered every haptic cue, because all haptic cues except Doubletime metronome turn on only when the subject slows down below $98 \%$ of $\omega_{\text {goal }}$. Thus, if the subject succeeded at staying above this speed, the haptic cue was not presented.

- Due to a software error, some trials were invalidated. Thus, we have a different $\mathrm{N}$ for some cues.
TABLE 3

Haptic cue occurrence and detection

\begin{tabular}{lccccc} 
& EBoost & $\begin{array}{c}\text { Asym } \\
\text { Res }\end{array}$ & Tap & $\begin{array}{c}2 x \\
\text { Metro }\end{array}$ & Vibrate \\
\hline Cue on, felt & 10 & 9 & 10 & 35 & 14 \\
Cue on, not felt & 23 & 4 & 2 & 1 & 5 \\
Not on, felt & 1 & 5 & 2 & 0 & 2 \\
Not on, not felt & 2 & 5 & 5 & 0 & 15 \\
Invalid trial & 0 & 13 & 17 & 0 & 0 \\
\hline Total & 36 & 36 & 36 & 36 & 36
\end{tabular}

Even when a haptic cue turned on, the subject may not have noticed it. That a cue was not consciously detected by a subject does not mean it did not affect his or her performance, however. Table 3 shows for each haptic cue the number of trials in which the haptic cue turned on and was detected by the subject, as evidenced by their answers on the questionnaires.

Only valid trials where the haptic cue turned on (the first two rows of Table 3) are considered in the analyses. This is reasonable because we are interested in knowing whether the cues are useful when they are encountered, not the frequency with which they are encountered.

\subsection{Quantitative results}

The results of the speed and power consistency calculations described in Sec. 6.4 are shown in Fig. 8. In this figure, the $\mathrm{p}$-value from the matched-pair $\mathrm{t}$-test is given above each cue on/cue off pair, and a $\sqrt{ }$ marks those that are statistically significantly different at $\mathrm{p}<0.05$. For reference, baseline speed ranged from $0.3109 \mathrm{~m} / \mathrm{s}$ to $0.6218 \mathrm{~m} / \mathrm{s}$ with an average of $0.4455 \mathrm{~m} / \mathrm{s}$, and baseline power ranged from $47.78 \mathrm{~W}$ to $190.12 \mathrm{~W}$ with an average of $98.75 \mathrm{~W}$.

The results of the secondary task analysis are shown in Fig. 9. Included on the right of this chart is the average number of misses over all trials and the average number of misses during "No Step", which is the average performance of subjects playing the video game without doing the stepping task. Comparing these two in a matchedpairs t-test, they did not prove statistically different.

\subsection{Qualitative results}

Selected results ${ }^{5}$ from the subject questionnaires are shown in Figs. 10, 11, 12, and 13. Displayed with each question is the p-value of a Kruskal-Wallis nonparametric ANOVA, which is the p-value informing whether there is a statistically significant difference between any of the five scores.

5. Only valid and interesting results are included in this manuscript. For an explanation of this and a complete list of questions and results, see [12]. 


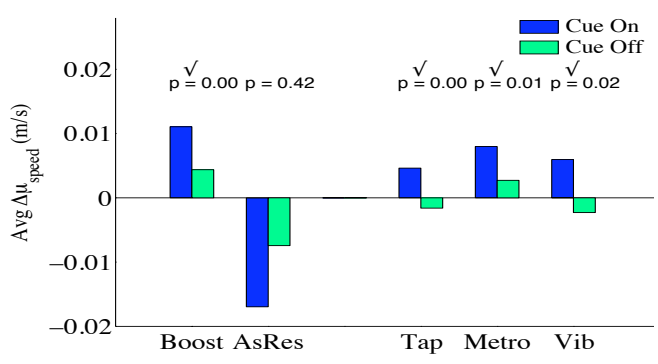

(a) $\bar{\Delta} \mu_{\text {speed }}$

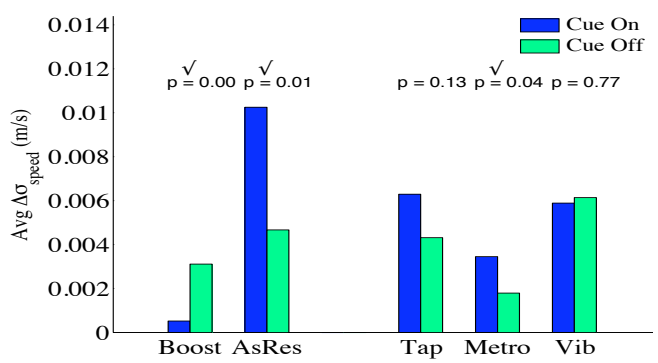

(c) $\bar{\Delta} \sigma_{\text {speed }}$

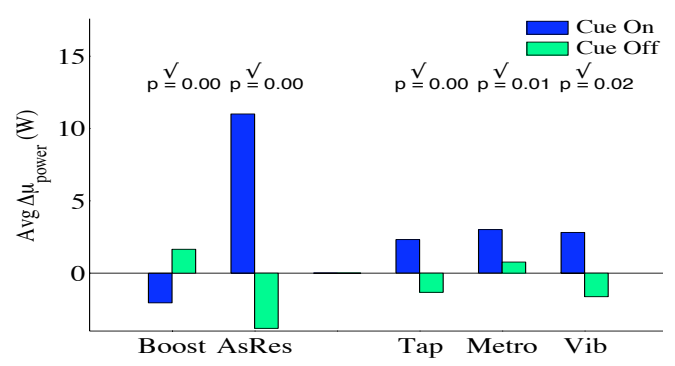

(b) $\bar{\Delta} \mu_{\text {power }}$

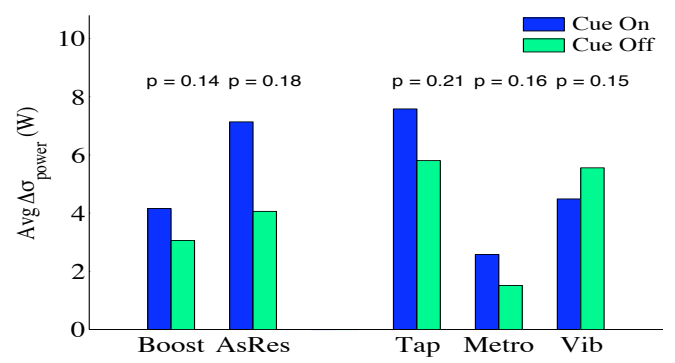

(d) $\bar{\Delta} \sigma_{\text {power }}$

Fig. 8. Average difference between the speed or power metric during the cue on/cue off period and during baseline

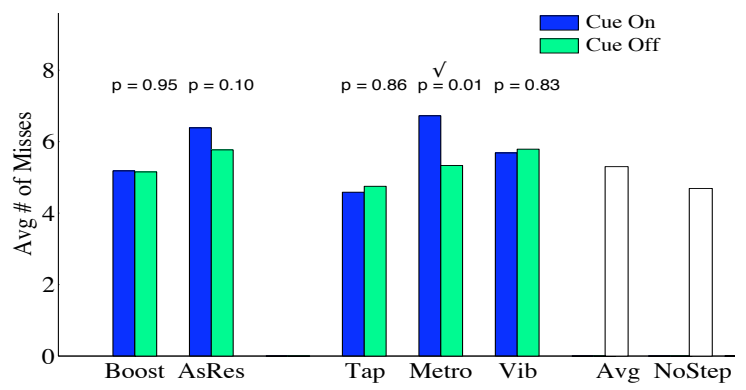

Fig. 9. Video game performance (zero is a perfect score)

\subsection{Results for each cue}

\subsubsection{E-Boost}

Considering the power metrics of Figs. 8(b) and 8(d), it can be seen that E-Boost was detrimental to the physical task of working out; on average, subjects exerted less power when using this cue than when not using it. This is due to the fact that E-Boost is assisting the user by contributing mechanical energy to the system. Even so, the cue did not reduce the subject's power variance.

The E-Boost cue often went undetected by subjects, as seen in Fig. 10. This is likely due to the cue turning on just below $98 \%$ of $\omega_{\text {goal }}$ and turning off once $\omega_{\text {goal }}$ is re-established, resulting in a speed change undetectable to the user. However, perhaps because of this, the EBoost cue allowed for some of the best performances on the video game (Fig. 9). Thus, if the task prioritization were slightly different, the E-Boost cue might be an appropriate choice of cue. For example, if we didn't care that the subject had high power output but only that he had good intra-period stepping consistency (Fig. 8(c)), or if performance on the video game was more important that performance on the physical task, E-Boost would be a very good haptic cue to use.

\subsubsection{Asymmetric Resistance}

Asymmetric Resistance had an obvious negative effect on stepping consistency, severely decreasing average speed (though not statistically significantly) and severely and statistically significantly increasing speed variance. As mentioned in Table 1, this cue results in a net increase in pedal motion resistance encountered by the user, thereby confounding the metrics of power for this cue. It also caused a problem, though not revealed by the data plots, in that several subjects got "stuck" at a slower speed; they knew they should speed up, but they could not overcome the additional resistance to stepping in order to get back to $\omega_{\text {goal }}$.

This cue likely doesn't have very high "resolution" when it comes to the subject's speed. That is, subjects can probably tell when their speed has dropped quite a bit, but this cue can't reliably alert them that their speed has slowed down just 2\% like Tapping or Vibration can.

Another problem with this cue is that it relies on the subject noticing a change in the damping resistance. That is, the subject must compare the resistance currently felt to the resistance that was being felt several seconds ago. This cue had five instances of subjects reporting to feel the cue when it never actually came on, more than any other cue type. It is likely that humans do not have significant precision in determining the amount of damping encountered during a motion. (Refer to work by Millman and Colgate [56] for more on perception of damping.) Therefore, if subjects are expecting that they might feel a change in damping and yet are not very good at detecting such a change, it is not unreasonable 


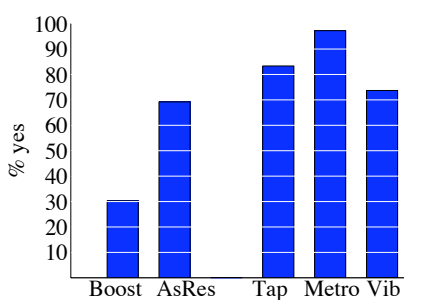

Fig. 10. Did you feel the cue? $(p=0.00)(\%$ of subjects that felt the cue when it was actually on)

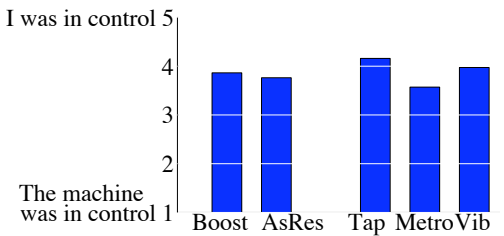

Fig. 11. How much did you feel "in control" of the stair climber? $(p=0.203)$

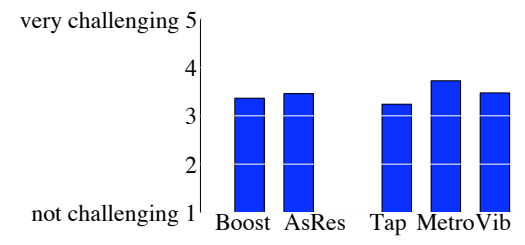

Fig. 12. Was completing both tasks mentally challenging? $(p=0.385)$

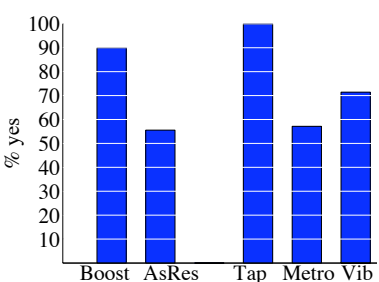

(a) helpful $(p=0.048)$

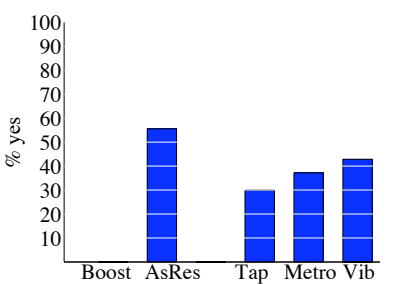

(b) annoying $(p=0.109)$

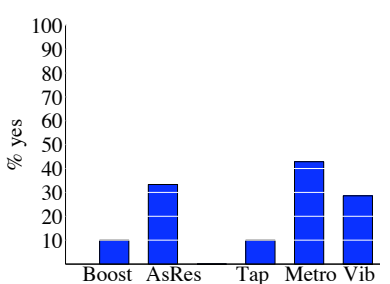

(c) confusing $(p=0.169)$

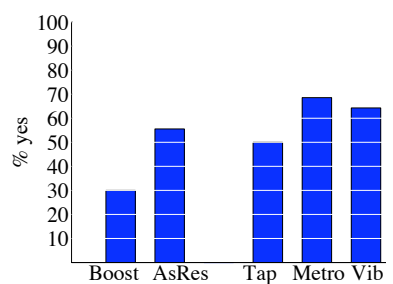

(d) distracting $(p=0.260)$

Fig. 13. Of the subjects who felt the cue when it was actually on, \% that found it...

to expect that some subjects will think they have encountered this change in damping when they have not. Since the theoretical purpose of the haptic cues in this experiment is to keep the subjects working out at a fast, consistent speed, it is not as bad to have false positives as it is to have subjects encounter the cue but not notice it (as is the case with the Vibration cue). Indeed, these false positives will simply keep the subject working out at an even higher rate. However, these false positives could cause problems depending on the task being undertaken.

\subsubsection{Tapping}

Tapping kept subjects stepping faster than $\omega_{\text {goal }}$ while minimizing total change in speed $\left(\Delta_{\mu}\right)$ more than any other cue. Change in standard deviation $\left(\Delta_{\sigma}\right)$ was higher than it was for most other cues, but the increase was not statistically significantly different between cue on and cue off. This cue did not have a statistically significant affect on video game performance, but subjects had their best average video game score using this cue.

The Tapping cue was the subjectively preferred cue of the subjects, and although it cannot be claimed without argument that it provided the best quantitative performance, it is apparent that it was at least good compared to other cues. It also has the advantage of being reliable since it was noticed by the highest percentage of subjects (Fig. 10) when it was on (except Doubletime Metronome, which was always on and noticed by all but one subject.)

\subsubsection{Doubletime Metronome}

Doubletime Metronome kept subject speed above $\omega_{\text {goal }}$, but it also increased variance. It was the only cue that had a statistically significant negative impact on video game performance. Perhaps this is because it is the only cue that is always on, continually fighting for the subject's attention.

Several subjects said that it was hard to follow the Doubletime Metronome and they thought they would have preferred a one-to-one beat. One subject who had a musical background said that beats of consistent intensity or accent are harder to follow than beats with changing intensity, especially so with the beat of this haptic cue which is only a two-count beat and is very fast. For example, it is easier to follow a beat such as $1,23,4,1,23,4$ than it is to follow a beat such as $1,2,1,2,1,2,1,2$, as this haptic cue is. Such a changing accent could be mimicked by changing the amplitude of the haptic cue every other beat.

This cue likely doesn't have very high "resolution". That is, even though we can sense a deviation in tapping of only $4 \mathrm{~ms}$ under controlled conditions [51], our ability to do so is probably much lower when a) the signal is coming through the feet, and b) there is significant mechanical noise. Therefore, this cue probably cannot regulate a subject's speed within $2 \%$ the way cues such as Tapping or Vibration can (where the subject knows for certain when he is not within $2 \%$ of $\omega_{\text {goal }}$.)

\subsubsection{Vibration}

The Vibration cue helped subjects maintain a speed above $\omega_{\text {goal }}$. It was the only cue that reduced variance in speed (other than E-Boost, which cannot be evaluated using velocity metrics), although not to a statistically significant extent. Performance on the mental task of playing the video game was not affected by this cue.

It is apparent that the Vibration cue may not be as salient as it needs to be, as evidenced by the $26 \%$ of subjects who did not notice the cue when it was on (Fig. 10). Using the Vibrating Pulses cue (see Table 2) 
instead of Vibration may have avoided this problem, or van Erp suggests that each subject should set his own vibration amplitude so as to ensure that it is noticeable by every subject [53]. However, it could simply be that a vibration on the foot is not a reliable means of haptic communication during an active task such as our exercise task. This could be due to mechanical noise of the stair climber masking the vibration cue, or because some subjects' shoe dampen the vibration more than others.

\section{Discussion}

Our results show that haptic communications can be effective even when presented on the legs, which are producing power-level forces and dealing with substantial haptic noise from the environment and internal sensations of exertion. They confirm that such haptic communications can assist an individual in completing a manual control task without taxing the visual channel that is necessary for many secondary tasks. Finally, they demonstrate that these communications need not be at the focus of the user's attention but instead can be presented as a peripheral sensation.

Overall, the tactile cues outperformed the kinesthetic cues in the exercise task. Both Vibration and Tapping made a statistically significant difference in keeping subjects stepping above their goal speed without being detrimental to the secondary task. Tapping was subjectively rated as the best cue, being noticeable and helpful without being too annoying, confusing, or controlling. The tactile cue of Doubletime Metronome also proved sufficient at keeping subjects stepping at a faster rate, but it negatively affected intra-period stepping consistency and secondary task performance. This may be due to its continuous presence, but we believe that other, more subtle continuous cues may still have merit and should be further investigated in future work. Also, our trials were much shorter than the average workout, and future work could explore the effect of longer exposure to the haptic cues during exercise.

The kinesthetic cues also had more side-effects than did the tactile cues. Although E-Boost helped subjects step above their goal speeds and was the only cue to provide a statistically significant improvement in intraperiod stepping consistency, it resulted in reduced subject power output, thereby undermining the exercise task. Asymmetric Resistance had a severe detrimental impact on both inter-period and intra-period stepping consistency. It may be possible to mitigate these effects by modifying the implementation of this cue.

\section{ACKNOWLEDGMENTS}

The authors would like to acknowledge National Science Foundation grant ECS 0433948 for support of this work.

\section{References}

[1] IXR, Inc. Exerscape software and DVDs. 1131 SW Long Creek Bend, OR 97702 http:/ / www.exerscape.com.

[2] Cateye Fitness. GameBike. 11116 Grader St. Dallas, TX 75238 http://www.cateyefitness.com/GameBike.

[3] InterAction Labs. XR Station. 12300-B Kiln Ct. Beltsville, MD 20705. http://www.ia-labs.com.

[4] G. Raphael, "Videogame workout blog." [Online]. Available: http://videogameworkout.com

[5] ICON Health and Fitness. iFit. 1500 South 1000 West, Logan, UT 84321. http://www.ifit.com.

[6] Yamaha Corporation of America. Bodibeat. 6600 Orangethorpe Ave. Buena Park, CA 90620. http://www.yamaha.com/bodibeat.

[7] G. Wijnalda, S. Pauws, F. Vignoli, and H. Stuckenschmidt, "A personalized music system for motivation in sport performance," Pervasive Computing, pp. 26-32, July-September 2005.

[8] F. Mertesdorf, "Cycle exercising in time with music," Perception and Motor Skills, vol. 78, no. 3 Pt 2, pp. 1123-1141, 1994.

[9] MYTRAK Health Systems, Inc. 3250 Ridgeway Dr. Suite 10 Mississauga, ON Canada L5L 5Y6. http://www.mytrakhealth.com.

[10] D Squared Technologies, Inc. TV Pedaler. P.O. Box 225, Jerico, NY 11753. http:/ / www.tvpedaler.com.

[11] PowerUp Fitness. The EnterTrainer Exercise Motivator. http://www.theentertrainer.com.

[12] A. R. Ferber, "Affecting exercise intensity through haptic communications," Master's thesis, Northwestern University, Evanston, IL, June 2007.

[13] A. R. Ferber, M. Peshkin, and J. E. Colgate, "Using haptic communications with the leg to maintain exercise intensity," in 16th IEEE International Workshop on Robot and Human Interactive Communication (RO-MAN '07), August 2007, pp. 292-297.

[14] A. Chan, K. MacLean, and J. McGrenere, "Learning and identifying haptic icons under workload," in First Joint Eurohaptics Conference and Symposium on Haptic Interfaces for Virtual Environment and Teleoperator Systems, 18 March 2005, pp. 432-439.

[15] H. S. Vitense, J. A. Jacko, and V. K. Emery, "Multimodal feedback: An assessment of performance and mental workload," Ergonomics, vol. 46, no. 1-3, pp. 68-87, 2003.

[16] C. Basdogan, C.-H. Ho, M. A. Srinivasan, and M. Slater, "An experimental study on the role of touch in shared virtual environments," ACM Transactions on Computer-Human Interaction, vol. 7, no. 4, pp. 443-460, December 2000.

[17] J. C. Roberts and K. Franklin, "Haptic glyphs (hlyphs) - structured haptic objects for haptic visualization," in First Joint Eurohaptics Conference and Symposium on Haptic Interfaces for Virtual Environment and Teleoperator Systems, March 18-20 2005, pp. 369-374.

[18] S. A. Wall and W. S. Harwin, "Quantification of the effects of haptic feedback during a motor skills task in a simulated environment," in 2nd PHANToM Users Research Symposium, 2000.

[19] A. F. Rovers and H. A. van Essen, "Guidelines for haptic interpersonal communication applications: An exploration of foot interaction styles," Virtual Reality, vol. 9, pp. 177-191, 2006.

[20] I. Oakley, S. Brewster, and P. Gray, "Can you feel the force? An investigation of haptic collaboration in shared editors," in Eurohaptics, 2001, pp. 54-59.

[21] M. K. O'Malley, A. Gupta, M. Gen, and Y. Li, "Shared control in haptic systems for performance enhancement and training," Journal of Dynamic Systems, Measurement, and Control, vol. 128, pp. 75-85, March 2006.

[22] E. Paulos, "Connexus: A communal interface," in Conference on Designing for User Experiences, San Francisco, CA, 2003, pp. 1-4.

[23] L. Rovers and H. van Essen, "Design and evaluation of hapticons for enriched instant messaging," Virtual Reality, vol. 9, pp. 177191, 2004

[24] A. F. Rovers and H. A. van Essen, "HIM: A framework for haptic instant messaging," in CHI 2004, Vienna, Austria, April 24-29 2004, pp. 1313-1316.

[25] A. Tang, P. McLachlan, K. Lowe, C. R. Saka, and K. MacLean, "Perceiving ordinal data haptically under workload," in 7th International Conference on Multimodal Interfaces. Torento, Italy: ACM, 2005, pp. 317-324.

[26] L. M. Brown, S. A. Brewster, and H. C. Purchase, "A first investigation into the effectiveness of tactons," in First Joint Eurohaptics Conference and Symposium on Haptic Interfaces for Virtual Environment and Teleoperator Systems, 2005, pp. 167-176. 
[27] A. Chang, S. O'Modhrain, R. Jacob, E. Gunther, and H. Ishii, "ComTouch: Design of a vibrotactile communication device," in Conference on Designing Interactive Systems, 2002, pp. 312-320.

[28] J. B. F. van Erp and H. A. H. C. van Veen, "Vibro-tactile information presentation in automobiles," Eurohaptics, 2001.

[29] T. A. Dingus, D. V. McGehee, N. Manakkal, S. K. Jahns, C. Carney, and J. M. Hankey, "Human factors field evaluation of automotive headway maintenance/collision warning devices," Human Factors, vol. 39, no. 2, pp. 216-229, 1997.

[30] M. Enriquez, O. Afonin, B. Yager, and K. Maclean, "A pneumatic tactile alerting system for the driving environment," in Proceedings of the 2001 workshop on perceptive user interfaces, 2001, pp. 1-7.

[31] C. Ho, H. Z. Tan, and C. Spence, "Using spatial vibrotactile cues to direct visual attention in driving scenes," Transportation Research Part F: Traffic Psychology and Behaviour, vol. 8, pp. 397-412, 2005.

[32] P. Griffiths and R. B. Gillespie, "Shared control between human and machine: Haptic display of automation during manual control of vehicle heading," in 12th International Symposium on Haptic Interfaces for Virtual Environment and Teleoperator Systems (HAPTICS '04), March 27-28 2004, pp. 358-366.

[33] M. Steele and R. B. Gillespie, "Shared control between human and machine: Using a haptic steering wheel to aid in land vehicle guidance," in Human Factors and Ergonomics Society 45th Annual Meeting, Minneapolis/St. Paul, MN, Oct 8-12 2001, pp. 1671-1675.

[34] D. A. Abbink, F. C. T. van der Helm, and E. R. Boer, "Admittance measurements of the foot during 'maintain position' and 'relax' tasks on a gas pedal," in IEEE International Conference on Systems, Man, and Cybernetics, 2004, pp. 2519-2524.

[35] M. Enriquez and K. E. MacLean, "Impact of haptic warning signal reliability in a time-and-safety-critical task," in 12th International Symposium on Haptic Interfaces for Virtual Environment and Teleoperator Systems (HAPTICS '04), March 27-28 2004, pp. 407- 414.

[36] P. Y. Li and R. Horowitz, "Control of smart exercise machines - Part I: Problem formulation and nonadaptive control," IEEE/ASME Transactions on Mechatronics, vol. 2, no. 4, pp. 237247, December 1997.

[37] _ _ "Control of smart exercise machines - Part II: Self-optimizing control," IEEE/ASME Transactions on Mechatronics, vol. 2, no. 4, pp. 248-258, December 1997.

[38] P. Li and R. Horowitz, "Intelligent control of an exercise machine," in $A M C$ ' 96 - MIE, 1996, pp. 271-276.

[39] P. Y. Li, J. Shields, and R. Horowitz, "Adaptive control of an exercise machine," IEEE, pp. 1278-1279, 1993.

[40] P. Y. Li and R. Horowitz, "Self-optimizing control: Application to smart exercise machines."

[41] R. Horowitz, P. Y. Li, and J. Shields, "Control of self-optimizing exercise machines," Annual Reviews in Control, vol. 24, pp. 201$213,2000$.

[42] J. Shields and R. Horowitz, "Adaptive step rate control of a stair stepper exercise machine." American Control Conference, June 1998, pp. 1058-1062.

[43] J. F. Shields, "Control of exercise machines: Theory and experiments," Ph.D. dissertation, University of California at Berkeley, Fall 1997.

[44] H. Kazerooni and M. Her, "A virtual exercise machine," IEEE, pp. 232-238, 1993.

[45] X. T. Zhang, D. M. Dawson, W. E. Dixon, , and B. Xian, "Experimental demonstration of an actuated exercise machine," in Proceedings of the 26th Annual International Conference of the IEEE EMBS, San Francisco, CA, September 2004, pp. 2707-2710.

[46] S. Motamarri, H. A. Malki, E. Barbieri, and E. J. Charlson, "Exercise machine controller design," in ISMCR, vol. B21, 2004, pp. $1-7$.

[47] R. C. Hayward and J. M. Hollerbach, "Implementing virtual stairs on treadmills using torso force feedback," in IEEE International Conference on Robotics and Automation, Washington, DC, May 2002, pp. 586-591.

[48] A. E. Minetti, L. Boldrini, L. Brusamolin, P. Zamparo, and T. McKee, "A feedback-controlled treadmill and the spontaneous speed of walking and running," Journal of Applied Physiology, vol. 95, pp. 838-843, 2003.

[49] C. D. Wickens, Engineering Psychology and Human Performance. Charles E. Merrill Publishing Company, 1984.

[50] A. M. Simon, R. B. Gillespie, and D. P. Ferris, "Symmetry-based resistance as a novel means of lower limb rehabilitation," Journal of Biomechanics, vol. 40, no. 6, pp. 1286-1292, July 142007.
[51] N. P. Lago and F. Kon, "The quest for low latency," in International Computer Music Conference, 2004, pp. 33-36.

[52] M. J. Enriquez and K. E. MacLean, "The hapticon editor: A tool in support of haptic communication research," in 11th Symposium on Haptic Interfaces for Virtual Environment and Teleoperator Systems, March 2003, pp. 356-362.

[53] J. B. F. van Erp, "Guidelines for the use of vibro-tactile displays in human computer interaction," in Eurohaptics, 2002.

[54] X. D. Pang, H. Z. Tan, and N. I. Durlach, "Manual discrimination of force using active finger motion," Perception \& Psychophysics, vol. 49, no. 6, pp. 531-540, June 1991.

[55] R. Margaria, Biomechanics and Energetics of Muscular Exercise. Clarendon Press, 1976.

[56] P. A. Millman and J. E. Colgate, "Effects of non-uniform environment damping on haptic perception and performance of aimed movements," in 4th Annual Symposium on Haptic Interfaces, November 1995.

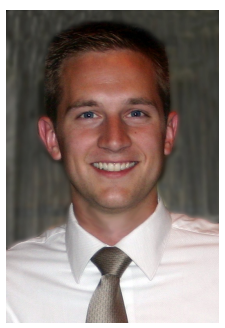

Aaron R. Ferber received his M.S. in Mechanical Engineering from Northwestern University in 2007 and his B.S. in Mechanical Engineering from Washington University in St. Louis in 2004. While at Northwestern University, he studied haptic communications and variable compliance haptic displays. He currently works as an Engineering Designer at IDEO, LLC in Chicago, IL, developing products and services from a humancentered design perspective.

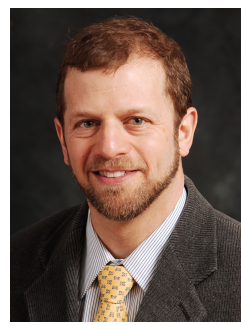

Michael Peshkin, Ph.D., is a Professor of Mechanical Engineering at Northwestern University. His research is in robotics and humanmachine interaction, and rehabilitation robotiics. He also works in novel actuators and sensors. Peshkin has co-founded three spin-offs: Mako Surgical, Inc. (image guided surgery), Cobotics, Inc. (assistive devices for materials handling) and Kinea Design, (engineering design for human.machine interface) Peshkin holds 15 patents, and is a co-inventor (with $\mathrm{J}$. Edward Colgate) of Cobots: collaborative robots for direct interaction with humans in a shared task.

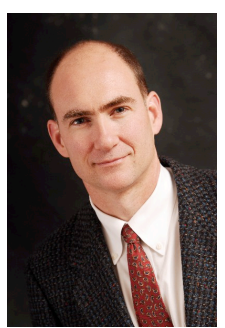

J. Edward Colgate J. Edward Colgate received the Ph.D. degree in mechanical engineering in 1988 from M.I.T. He subsequently joined Northwestern University in Evanston, Illinois, where he is currently the Pentair-Nugent Professor in the Department of Mechanical Engineering. Dr. Colgate's principal research interest is humanrobot interaction. He has worked extensively in the areas of haptic interface and teleoperation, and he, along with collaborator Michael Peshkin, is the inventor of a class of collaborative robots known as cobots. He has served as an associate editor of the Journal of Dynamic Systems, Measurement and Control and the IEEE Transactions on Robotics and Automation, and he is the founding Editor-inChief of the IEEE Transactions on Haptics. In addition to his academic pursuits, Dr. Colgate is a founder of Stanley Cobotics, the leading supplier of intelligent ergonomic assist devices to the industrial marketplace and of Kinea Design, a start-up devoted to rehabilitation robotics and advanced mechatronics. Dr. Colgate is currently the co-Director (with Don Norman) of the Segal Design Institute at Northwestern University. In this capacity, he is directing a new Master of Science in Engineering Design and Innovation, which combines graduate-level engineering courses with a broad exposure to human-centered design. 